\title{
Managing for the Long-term - Strategies for Initiating and Maintaining Successful Continuous Dopaminergic Stimulation Treatment
}

\author{
Tove Henriksen, ${ }^{1}$ Per Odin, ${ }^{2}$ Jens Volkmann ${ }^{3}$ and Angelo Antonini ${ }^{4}$
}

\begin{abstract}
1. Consultant, Movement Disorder Clinic, Bispebjerg Hospital, University Hospital of Copenhagen, Denmark; 2. Chairman, Department of Neurology, Klinikum-Bremerhaven, Bremerhaven, Germany and Professor, Department of Neurology, Lund University Hospital, Lund, Sweden; 3. Chairman, Department of Neurology, University Hospital Würzburg, Germany; 4. Director, Parkinson Unit, IRCCS San Camillo, Venice, and University of Padua, Padua, Italy
\end{abstract}

\begin{abstract}
There are many factors that may influence treatment choice in patients with Parkinson's disease. While 'inclusion' criteria include the clinical need for short- and long-term improvement, there are some well defined contraindications to continuous dopaminergic stimulation (CDS) therapies, such as age, cognitive impairment, psychosis or severe orthostatic hypotension. These contraindications vary between CDS therapies and can help guide the choice of treatment. Patient preferences and the practicalities of the treatment modality also play an important part in the decision-making process. Treatment selection algorithms have been developed and presented, but they require refining and need to be expanded to include guidance on management decisions - for example, when initial CDS therapy fails. While more trial data are gathered and as management algorithms evolve, case studies continue to provide important information on practical aspects of CDS therapies.
\end{abstract}

\section{Keywords}

Parkinson's disease, continuous dopaminergic stimulation, deep brain stimulation, subcutaneous apomorphine infusion, levodopa/carbidopa intestinal gel infusion, treatment selection algorithm

Disclosure: Tove Henriksen has a consultancy agreement with Medtronic and an advisory board agreement with Abbott. Per Odin has received honoraria for lectures and participation in advisory boards from Boehringer Ingelheim, Abbott, Cephalon, Union Chimique Belge (UCB) and GlaxoSmithKline (GSK). Jens Volkmann has no conflicts of interest to declare. Angelo Antonini has received honoraria for consulting services and symposia from Abbott, Boehringer Ingelheim, GSK, Lundbeck, UCB, Novartis and Merck-Serono.

Acknowledgements: The V International Forum on Parkinson's Disease (Helsinki, Finland, 6-7 May 2011) was funded by an unrestricted educational grant from Abbott. Abbott funded the development of this supplement by ESP Bioscience (Crowthorne, UK). Emily Chu and Nicole Meinel of ESP Bioscience provided medical writing and editorial support to the authors in the development of this publication. Abbott had the opportunity to review and comment on the publication's content; however, all decisions regarding content were made by the authors. Tove Henriksen would like to thank Narissah Bryndum, Parkinson's disease nurse at Bispebjerg Hospital. Received: 22 June 2012 Accepted: 9 July 2012 Citation: European Neurological Review, 2012;7(Suppl. 1):31-5

Correspondence: Tove Henriksen, Movement Disorder Clinic, Bispebjerg Hospital, University Hospital of Copenhagen, Bispebjerg Bakke 23, 2400 Copenhagen, Denmark. E: tovehenriksen@dadlnet.dk

\section{Options for Continuous Dopaminergic Stimulation Therapy}

In patients with advanced Parkinson's disease (APD), worsening motor symptoms due to motor response complications and dyskinesia (exacerbated by erratic gastric emptying associated with peroral therapy) may be treated optimally by switching to continuous dopaminergic stimulation (CDS) therapy. There are three CDS therapy options widely available for the treatment of APD:

- levodopa/carbidopa intestinal gel (LCIG) infusion;

- subcutaneous apomorphine (APO) infusion; and

- deep brain stimulation (DBS; primarily of the subthalamic nucleus [STN]), which is not strictly a CDS treatment but has similar effects.

But how do we choose between these treatments for a given Parkinson's disease (PD) patient? Currently, there are no large-scale, randomised, placebo-controlled trials directly comparing the three CDS treatment options. We must therefore rely on indirect comparisons and a growing body of clinical experience to judge the appropriateness of each CDS therapy for individual patients.

\section{Factors to Aid Treatment Choice}

\section{Inclusion and Exclusion Criteria}

We can use various criteria to decide whether a patient is appropriate for a given treatment, including age, cost, practicalities (e.g., feasibility of surgery or availability of a multidisciplinary care team), cognitive impairment or psychosis (see Figure 1). APO infusion is the 'simplest' treatment in terms of administration, but may be slightly less effective in treating motor complications than DBS or LCIG infusion. Furthermore, different CDS treatments appear to improve different non-motor symptoms (NMS). If there is a 24-hour clinical need, DBS may be the better choice and, in the long-term, may be cheaper than pump therapies. If the patient is aged $>70$ years, they may not be a good candidate for DBS given the risks of surgery. In addition, cognitive impairment or severe psychosis may preclude all CDS therapies, but especially DBS. Patients with impulse control disorder, hypersexuality or 
Figure 1: Characteristics of the Typical Patient for Each of the Three Continuous Dopaminergic Stimulation Therapies
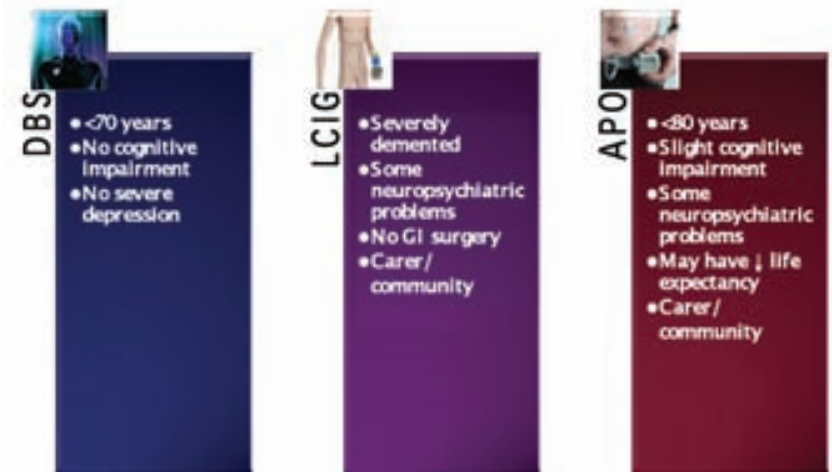

$A P O=$ subcutaneous apomorphine infusion; $D B S=$ deep brain stimulation $G l=$ gastrointestinal; $L C I G=$ levodopa/carbidopa intestinal gel infusion

severe orthostatic hypotension may not be good candidates for APO infusion. Co-morbidities such as gastrointestinal problems may make percutaneous endoscopic gastrostomy (PEG) fitting for LCIG infusion problematic. Any other disease that has an effect on life expectancy may make the neurologist more reluctant to suggest surgery, given the added risk of unpleasant side effects that may lead to death or severe morbidity.

\section{Safety Profiles and Patient Preferences}

Patients' own preferences can influence treatment choice when al three CDS therapies are appropriate, which is more likely to be the case in young patients. It is extremely important for the neurologist to ensure that patients and their care-givers are thoroughly informed of the details of each of the CDS therapies, including associated side effects and complications. This would help ensure that they make the best decision for themselves and do not have unrealistic expectations. For example, presenting patients with the pump so they are familiar with its size, or showing them the needle and PEG tube and drawing their attention to the need to change these every morning, may make them better appreciate the reality of the treatment.

The side effect profiles associated with each of the three CDS therapies may assist the neurologist and patient/care-giver in their choice of treatment. Patient preferences are often related to the side effect profile of the treatments, with adverse events such as worsening dysarthria (experienced with DBS by $43 \%$ of patients in a five-year follow-up study'), orthostatic hypotension (experienced with APO infusion by $7 \%$ of patients ${ }^{2}$ ), depression (experienced with DBS by $26 \%$ of patients'), apathy (experienced with DBS by $13 \%$ of patients ${ }^{1}$ ), hallucinations (experienced with APO infusion by $14 \%$ of patients ${ }^{2}$ ), increased libido (experienced with APO infusion by $5 \%$ of patients ${ }^{2}$ ), sedation (experienced with APO infusion by $20 \%$ of patients ${ }^{2}$ ), abdominal wall nodules (experienced with APO infusion by up to $80 \%$ of patients, ${ }^{2}$ but a minor problem in most patients), infection (experienced with LCIG infusion by $10 \%$ of patients ${ }^{3}$ ) and peritonitis (experienced with LCIG infusion by $4 \%$ of patients ${ }^{3}$ ). The side effect profile that patients are willing to accept depends on their individual circumstances. For example, a person with APD whose professional career depends on oral communication (e.g., an actor) may find the risk of dysarthria with DBS unacceptable and so may not opt for DBS. Indeed, it could be argued that patients who opt for DBS may be greater risk takers. However, many factors come into play in an individual's preference for a particular CDS therapy. Moreover, once a patient has started CDS therapy, the therapy must be optimised both in the short- and long-term.

\section{Other Factors}

Other factors that must be considered when deciding which is the best therapy include whether the treatment centre has experience of performing that particular therapy, whether it has a multidisciplinary care team, whether the patient has a care-giver at home and whether the therapy is reimbursed. It is extremely important that the neurologist is familiar with the patient's home environment and that they obtain this information through direct discussion with the patient rather than by taking a patient history from the referral centre.

\section{Optimising Continuous Dopaminergic Stimulation Therapy in the Short-term}

A patient's understanding of, and adherence to, medication can be regarded as the sixth vital sign when evaluating a patient's medical status. ${ }^{4}$ Adherence to medication is extremely important when dealing with complex treatments such as CDS therapies. But what can we do to improve adherence? In the short-term, it is crucial to provide as much information as possible before a decision is made on which CDS therapy to choose. Patients and their care-givers must be informed about the expected effects, side effects, complications, practicalities and long-term effects of the therapies. They need to have realistic expectations about the therapies - otherwise they may become disheartened with the outcome, which may negatively impact their adherence to treatment. The clinic should be specialised in the chosen CDS treatment, and preferably in all three CDS therapies. Experience in all three CDS therapies as well as dealing with possible side effects/complications will result in better patient selection. In addition, PEG surgery should only be performed by a specialised team in order to reduce risks.

\section{Optimising Continuous Dopaminergic Stimulation Therapy in the Long-term}

While there is no evidence of neuroprotection, the available evidence shows that the effect of all three CDS therapies continues over a period of five years. ${ }^{5-13}$ There is no evidence of tolerance for any of the three CDS treatments; in fact, there is evidence that the doses on pump therapies can be lowered over time, and that the therapeutic window can be reopened with all three CDS treatments.

Factors that may be important for optimising therapy in the long-term are listed below.

- Ensuring easy access to the outpatient clinic for:

- titrating medication (especially at the start of treatment);

- adjusting dose; and

- treating adverse effects as soon as possible.

- Having only one PD nurse, and ideally only one movement disorder clinician, assigned to each patient.

- Giving a telephone contact for when help is needed out-of-hours.

- Offering the possibility of sub-acute consultations or admissions as necessary.

- Providing continuous support to care-giver

- Including care-giver in outpatient clinic visits to get greater insight into patient's home life.

- Keeping in close contact with general practitioner and community nurse.

- Offering advice/assistance when the patient:

- is admitted at another hospital; and/or

- needs surgery. 


\section{Managing Problems in Continuous Dopaminergic Stimulation Therapy}

Trouble-shooting may be required during the course of CDS therapy. With DBS, there is a risk of infection and displacement of electrodes, which can elicit sudden side effects such as dystonia, pain, diplopia and dysarthria. There may also be arrest of the implanted pulse generator. With LCIG infusion, there may be pump failure or problems with the inner tubing such as disconnection, obstruction or dislocation with secondary migration in the intestine. With APO infusion, there may be pump failure or formation of subcutaneous nodules in the abdominal wall, which may become infected and require immediate treatment.

In addition, side effects may emerge with CDS therapy that may need to be managed. For example, motor fluctuations may develop with LCIG infusion, which can be managed with sustained-release (SR) agonist formulations, amantadine or a change in diet. In other cases, side effects may necessitate switching to a different CDS therapy. For example, freezing of gait and severe painful dystonia that can develop with DBS may require switching to LCIG infusion; APO infusion is another treatment alternative for DBS-associated dystonia. APO infusion carries a risk of psychosis, which may call for changing to LCIG infusion therapy As more trial data are gathered, case studies continue to provide important information on practical aspects of CDS therapies and how to manage some of these problems.

\section{Case 1 - Trouble-shooting During Apomorphine Infusion Therapy in a Patient with a Good Response to this Therapy \\ Patient History}

This male patient started experiencing symptoms, including a tendency to muscle fatigue in the left upper extremity and neck pain, in 1997 at the age of 47. His gait was stooped and he had a slight dysarthria. In 2001, he developed a slight resting tremor of the left hand and was diagnosed with idiopathic PD. This was initially managed with benzeraside/levodopa 25/100 mg three times daily, which achieved a very good motor response. In 2003, he developed motor fluctuations and 'wearing off', which was treated by adding entacapone $200 \mathrm{mg}$ six times daily to his regimen. The patient experienced side effects, including diarrhoea and severe weight loss, so entacapone was stopped. Cabergoline was added to his regimen and gradually increased from 2 to $6 \mathrm{mg}$ once daily, because of motor symptoms, rigidity and bradykinesia, and benserazide/levodopa 25/100 mg was increased to six doses per day. In 2004, the patient developed a tendency to experience hyperkinesias and had dystonic pain in the neck area when in an 'off' state. He became increasingly sensitive to the influence of meals on the effect of his medication (a challenge when he was taking drugs six times daily). In 2006, the number of benserazide/levodopa doses was increased to seven per day and entacapone $200 \mathrm{mg}$ was again added to his regimen, but this time taken three times daily, following which the patient did not experience any diarrhoea. Echocardiography revealed that there was possible valvular fibrosis and mitral valve insufficiency. Consequently, cabergoline was replaced with pramipexole $0.7 \mathrm{mg}$ three times daily. This increased nuchal rigidity and motor fluctuations.

\section{Subsequent Treatment}

The patient was subsequently offered advanced therapies: either DBS, LCIG infusion or APO infusion. Given his 'off' periods, his preference was for pen-injected apomorphine treatment and he received add-on pen-injected apomorphine of $5 \mathrm{mg}$ in the morning, $4 \mathrm{mg}$ in the afternoon and an additional $4 \mathrm{mg}$ at night (up to a maximum of five injections a day). In 2007, the patient experienced night-time problems with dystonia in the lumbar region and left leg, as well as problems with mobility in the morning and problems with working. At this time, his therapy was benserazide/levodopa 25/100 mg six times daily, entacapone $200 \mathrm{mg}$ three times daily, plus pen-injected apomorphine administered approximately five times daily (4 mg per injection).

Around this time, he partook in a solo fishing expedition. He forgot to take his peroral therapy with him and so had to cope with the apomorphine pen alone. He experienced no hyperkinesias or tremor and, given this positive experience, he subsequently asked for APO infusion treatment. From then on he received:

- APO pump $5.25 \mathrm{mg} / \mathrm{h}$ applied for $16 \mathrm{~h} /$ day;

- benserazide/levodopa 25/100 mg four times daily;

- entacapone 200 mg three times daily;

- benserazide/levodopa SR 25/100 mg once daily; and

- domperidone $10 \mathrm{mg}$ three times daily.

\section{Outcomes of Continuous Dopaminergic}

Stimulation Therapy

The APO infusion therapy provided good motor control with very few fluctuations and little neck pain. Clinically, there were virtually no signs of PD. The patient used the pump in the daytime only. He used the apomorphine pen in the morning and when he was called out at night to retrieve a truck, in order to obtain enough dexterity to start the APO pump. He went hunting and fishing and was able to drive long-haul to the northern part of Sweden.

In 2009, the patient started to develop subcutaneous nodules with small ulcerations in the abdominal skin in spite of weekly ultrasound treatment. It was decided to change his needle from the steel needle he had been using so far to $\mathrm{Cleo}^{\circledR}$, a Teflon ${ }^{\circledR}$ needle that causes less irritation of the skin. This resulted in the irritation and ulcers completely disappearing. In 2010, he experienced difficulty handling the pump at night when he was in the 'off' state due to reduced manual dexterity. This was a problem given that he was often called to retrieve broken-down trucks during the night. To solve this, the pen system was changed to a pumpfill system, which demands less manual dexterity as it is a pre-filled syringe system.

\section{Conclusions}

By using the experience of a multidisciplinary team to trouble-shoot, it was possible to keep the patient on APO infusion treatment, allowing him to continue working and pursue his hobby of fishing. such trouble-shooting was only possible thanks to the in-depth experience of the team at the treatment centre, and we suggest that this is a prerequisite for optimal CDS therapy use.

\section{Case 2 - Managing a Patient with Motor Fluctuations and Depression with a Combination of Levodopa/Carbidopa Intestinal Gel Infusion and Dopamine Agonist}

Patient History

This female patient born in 1962 was diagnosed with PD in 1996 at the age of 34 years. She presented with hypokinetic-rigid symptomatology as well as a pronounced tendency to anxiety and depression. She was married and lived in a house in the countryside. In 2002, being treated with five doses of levodopa/benserazide $100 \mathrm{mg} / 25 \mathrm{mg}$ daily and three doses of pramipexole $0.35 \mathrm{mg}$ daily, she experienced the first 'off' fluctuations, which were followed in 2003 by dyskinesias. The 'off' 


\section{The Parkinson's Disease Virtual Clinic}

periods were with moderate motor symptoms, but pronounced anxiety and depressive thoughts. This patient was managed initially as follows:

- pramipexole $0.7 \mathrm{mg}$ three times daily;

- selegiline $10 \mathrm{mg}$ once daily;

- Ievodopa/carbidopa 100/25 mg six times daily; and

- citalopram 40 mg once daily.

In order to improve her depression and anxiety, her pramipexole medication was raised to $3.15 \mathrm{mg}$ daily, with no positive effect. She was admitted to psychiatric departments for long periods and was administered various antidepressive and anxiolytic drugs and mood stabilisers, with no major improvement. In addition, psychotherapy had a limited effect.

\section{Outcomes of Continuous Dopaminergic Stimulation Therapy}

The patient was subsequently offered advanced pump therapy. LCIG infusion ( $1.7 \mathrm{ml} / \mathrm{h} 16 \mathrm{~h} /$ day) was administered, with $2 \mathrm{ml}$ on demand. concomitant peroral medication was administered as follows:

- pramipexole $1.05 \mathrm{mg}$ in the evening;

- levodopa/carbidopa SR 100/25 mg in the evening; and

- escitalopram 20 mg once daily.

This regimen reduced 'off' time by $75 \%$ (to approximately $1 \mathrm{~h} /$ day) reduced the frequency of dyskinesias and improved non-motor fluctuations, anxiety and depression in the daytime. However, with the optimum LCIG infusion dose for reducing dyskinesias, depression and anxiety reappeared. With slight-to-moderate dyskinesias, there was no depression or anxiety. Consequently, a higher dose of LCIG was infused and some dyskinesias were tolerated. With this strategy, the patient had no daytime depression/anxiety. As a result of night-time PD symptoms, sleep was suboptimal and the patient experienced depressive thoughts and anxiety at night. Consequently, LCIG was infused over 24 hours and concomitant peroral medication included pramipexole (1.05 $\mathrm{mg}$ in the evening) and escitalopram (20 mg daily). There was a strong improvement in sleep and night-time symptomatology. The patient subsequently stopped taking pramipexole; LCIG was thus given as monotherapy and substantially higher doses were necessary, which meant that depressive thoughts increased once again. Consequently, pramipexole $1.05 \mathrm{mg}$ in the evening was reintroduced. As a result, LCIG dose requirements returned to normal levels and depressive thoughts diminished.

\section{Conclusions}

This patient case highlights that LCIG infusion can have a good effect on 'off' period-related NMS such as depression and anxiety. It also illustrates that higher LCIG doses may be necessary to treat the psychiatric symptomatology compared with the motor symptoms, and that dopamine agonists may be valuable as co-medication in some patients.

\section{Case 3 - Managing Psychosis and Social Maladjustment after Deep Brain Stimulation} Patient History

This 52-year-old former police officer had a 15-year history of akinetic-rigid PD and had had to retire early because of the condition. He presented as an emergency case in 2003, and his chief complaints were motor fluctuations (approximately $75 \%$ daily 'off' time and $25 \%$ daily 'on' time); during 'off' time, he was immobile due to severe gait disorder and eyelid opening apraxia; during 'on' time, he experienced dystonic dyskinesia with blepharospasm, causing functional blindness; and he had frequent, fearful delusions (he saw and felt rats running over his face at night), causing major distress. He essentially had no functional 'on' time. His PD was initially managed with:

- levodopa/carbidopa 100/25 mg six times daily;

- entacapone 200 mg once daily;

- cabergoline $2.5 \mathrm{mg}$ once daily;

- amantadine $250 \mathrm{mg}$ per day (split into one $100 \mathrm{mg}$ and one $150 \mathrm{mg}$ dose); and

- clozapine 25 mg once daily.

In 2003, LCIG infusion was not available in Germany and APO infusion and DBS were contraindicated due to the patient's ongoing psychosis. Thus, given that the first priority was to alleviate his psychosis, the patient's regimen was reverted to levodopa monotherapy and amantadine and cabergoline were withdrawn. His treatment regimen was levodopa/entacapone plus clozapine $50 \mathrm{mg}$ at night-time.

At a three month follow-up visit, the patient had more pronounced motor fluctuations. He was bedridden during 'off' periods (approximately $50 \%$ of the day), had moderate choreatic dyskinesia and had frequent falls during 'on' periods. He experienced no delusions and had normal cognitive function (Mattis dementia scale 138/140). He had an excellent levodopa response - Unified Parkinson's Disease Rating Scale Part III 'off': 56 and 'on': 23. Subsequently, he requested advanced treatment in order to regain functional motor capacities and to allow him to continue living with his family (his wife wanted him to move to a nursing home).

\section{Outcomes of Continuous Dopaminergic Stimulation Therapy}

For this patient, APO infusion was not an option given his tendency to respond to oral dopamine agonists with psychosis. DBS of the globus pallidus interna would have required that the patient remained on substantial amounts of medication due to his tendency to psychosis. A clinical decision was made to administer STN-DBS, as this would allow the amount of oral medication to be substantially lowered, thereby having a positive effect on confusional states and providing more continuous 'on' periods.

Six months after starting STN-DBS, the patient displayed severe stimulation-induced dyskinesia and it was necessary to stop all oral medication. He stayed in hospital for eight weeks in order to allow titration of the medication. He consequently experienced apathy caused by levodopa withdrawal, and a fall during rehabilitation resulted in an unstable fracture of the lumbar vertebra and subsequent bed-rest for six weeks. Twelve months after starting STN-DBS, the patient experienced no more 'off' periods and had permanent but very mild stimulation-induced dyskinesia. He had no more falls. However, despite the motor benefits, the patient was not happy. His wife divorced him during a prolonged rehabilitation period and he could not return home. He moved to a nursing home, had no friends and was unable to work.

Five years after initiating STN-DBS, he continues without dopaminergic medication. He experiences no 'off' periods or dyskinesia. He shows progression of axial motor symptoms, including mild postural imbalance and start hesitation, and uses a walker for longer distances. He has mild apathy, but lives in his own apartment and has a girlfriend. 


\section{Conclusions}

For this patient, STN-DBS was initially contraindicated due to pre-operative psychosis, but this condition can be managed. If controlled, previous psychosis does not exclude patients from surgery. In addition, the patient had unrealistic treatment goals and a difficult social environment. The case suggests that APD therapies should be applied earlier to prevent social maladjustment.

\section{Case 4 - Levodopa/Carbidopa Intestinal Gel Infusion in a Relatively Young Patient in whom Deep Brain Stimulation was Contraindicated Due to Mild Cognitive Impairment, Orthostatic Hypotension and Other Factors \\ Patient History}

This 54-year-old male experienced the onset of PD in 2003 at the age of 46 . His first symptoms were fatigue and slowness, which improved with levodopa (100 mg three times daily) and ropinirole (12 mg/day). Five years later, he developed motor fluctuations with severe 'off' disability. Most of the 'off' periods were poorly responsive to additional levodopa administrations due to erratic gastric emptying. Ropinirole led to hypersexuality and gambling behaviours and was thus stopped. His symptoms were managed with:

- levodopa/carbidopa tablets $100 \mathrm{mg}$ every three hours, six times daily;

- levodopa/benzeraside soluble $100 \mathrm{mg}$, up to four times daily;

- levodopa/carbidopa extended-release 200 mg, one tablet before bedtime; and

- rotigotine $8 \mathrm{mg}$ daily.

The patient had three hours of 'on' time with moderate dyskinesias and six hours of 'off' time per day. He had severe sleep fragmentation with nocturia (approximately four times per night). It was decided that a change in therapy was needed to improve his symptoms as he wanted to become clinically independent.

\section{Outcomes of Continuous Dopaminergic Stimulation Therapy}

The patient was subsequently evaluated for DBS. He had a mini-mental state examination score of 26 , with moderate deficits in frontal lobe function and memory as indicated by the mild cognitive impairment multi-domain score - which is a contraindication to DBS. His sleep disturbances (rapid eye movement sleep behaviour disorder [RBD]) were evident during hospital admission and he had severe orthostatic hypotension. As a result of his evaluation, he was prescribed LCIG infusion. Given that his stomach was an unusual shape (which explained why he had problems with gastric emptying), he required a jejunoscopy. After initiating LCIG infusion, his regimen was as follows:

- LCIG infusion (2.8 ml/h) from 07:00 to 22:00 (728 mg levodopa/day); given his sleep disturbances, 24-hour infusion was not appropriate;

- levodopa/carbidopa (2 $\mathrm{ml}$ additional dose at a maximum of three doses daily):

- rotigotine (8 mg daily from 22:00 to 08:00); and

- clonazapam (0.5 mg at bedtime) for the RBD

He has now been receiving LCIG infusion for six months and his symptoms have improved substantially, with a reduction of the number of hours spent in 'off' from six to three. Continual evaluation of his cognitive and autonomic function is required.

\section{Conclusions}

STN-DBS was not considered for this patient given the presence of subtle cognitive changes. Equally, APO infusion was not considered as the first choice of therapy because of the patient's history of impulse control disorders. This case demonstrates that LCIG infusion is beneficial in the management of patients presenting with mild cognitive abnormalities and absorption problems with oral levodopa.

\section{Summary}

Before selecting a specific CDS therapy for a given patient, it is important to consider:

- the need for a thorough evaluation of the patient;

- the need to provide thorough information to the patient and care-giver;

- the likelihood of patient adherence to the particular CDS therapy;

- the availability of a multidisciplinary team that has experience of the chosen CDS therapy;

- the availability of that therapy at the treatment centre (ideally, the centre should have experience of all three CDS treatments); and

- the need for close follow-up.

To date, there have been no randomised, placebo-controlled investigations directly comparing DBS, APO infusion and LCIG infusion. However, case studies continue to provide important information on the practical aspects of CDS therapies. There are a number of factors that can be taken into account when deciding which is the best CDS therapy for a given patient, such as: expected benefits of therapy, duration of expected benefits, side effect profile, price, and how long the patient is otherwise expected to live. Treatment selection algorithms have been developed, ${ }_{14}^{14}$ but they require refining and need to be expanded to include information on what management decisions are needed - for example, what to do when initial CDS therapy fails.
1. Schüpbach WM, Chastan N, Welter ML, et al., Stimulation of the subthalamic nucleus in Parkinson's disease: a 5 year follow up, J Neurol Neurosurg Psychiatry, 2005;76:1640-4.

2. Odin P, Hagell P, Shing M (eds), Apomorphine in Parkinson's Disease, Bremen: Uni-Med Verlag AG, 2008.

3. Devos $D$, Patient profile, indications, efficacy and safety of duodenal levodopa infusion in advanced Parkinson's disease, Mov Disord, 2009:24:993-1000.

4. Rosenow EC 3rd, Patients' understanding of and compliance with medications: the sixth vital sign? Mayo Clin Proc, 2005;80:983-7.

5. Moro E, Lozano AM, Pollak P, et al., Long-term results of a multicenter study on subthalamic and pallidal stimulation in Parkinson's disease, Mov Disord, 2010;25:578-86.

6. Simonin C, Tir M, Devos D, et al., Reduced levodopa- induced complications after 5 years of subthalamic stimulation in Parkinson's disease: a second honeymoon J Neurol, 2009;256:1736-41.

7. Stocchi F, Vacca L, De Pandis MF, et al., Subcutaneous continuous apomorphine infusion in fluctuating patients with Parkinson's disease: long-term results, Neurol Sci, 2001;22:93-4.

8. Kanovský $\mathrm{P}$, Kubová D. Bares $\mathrm{M}$, et al., Levodopa-induced dyskinesias and continuous subcutaneous infusions of apomorphine: results of a two-year, prospective follow-up, apomorphine: results of a

Mov Disord, 2002;17:188-91.
Stocchi F, Ruggieri S, Vacca L, Olanow CW, Prospective randomized trial of lisuride infusion versus oral levodopa in patients with Parkinson's disease, Brain, 2002;125:2058-66.

10. Manson AJ, Turner K, Lees AJ, Apomorphine monotherapy in the treatment of refractory motor complications of
Parkinson's disease: long-term follow-up study of 64 patients, Mov Disord, 2002;17:1235-41.

11. Nilsson D, Nyholm D, Aquilonius SM, Duodenal levodopa infusion in Parkinson's disease - long-term experience, Acta Neurol Scand, 2001:104:343-8.

12. Katzenschlager R, Hughes A, Evans $A$, et al., Continuous subcutaneous apomorphine therapy improves dyskinesias in Parkinson's disease: a prospective study using singlein Parkinson's disease: a prospective study
dose challenges, Mov Disord, 2005;20:151-7.

13. Nyholm D, Lewander T, Johansson A, et al., Enteral levodopa/carbidopa infusion in advanced Parkinson disease: long-term exposure, Clin Neuropharmacol, 2008;31:63-73.

14. Antonini $A$, Tolosa $E$, Apomorphine and levodopa infusion therapies for advanced Parkinson's disease: selection criteria and patient management, Expert Rev Neurother, 2009;9:859-67. 